\title{
A Case of Bruxism-Induced Otalgia
}

\author{
Se-Hyung Kim \\ Department of Otorhinolaryngology-Head and Neck Surgery, Jeju National University School of Medicine, Jeju, Korea
}

$\begin{array}{ll}\text { Received } & \text { May 16, 2016 } \\ \text { Revised } & \text { June 13, 2016 } \\ \text { Accepted } & \text { June 16, 2016 }\end{array}$

Address for correspondence Se-Hyung Kim, MD, PhD

Department of OtorhinolaryngologyHead and Neck Surgery,

Jeju National University

School of Medicine,

15 Aran 13-gil, Jeju 63241, Korea

Tel +82-64-717-1720

Fax +82-64-717-1131

E-mail meddoc98@gmail.com
Here, the author presents a case of bruxism-induced otalgia in a 29-year-old female patient. The pain was sharp and penetrating in character. It was usually worse in the morning and frequently radiated to the right temporal area. She had received unsuccessful medical treatments for migraine headache. The otoendoscopic examination revealed a normal tympanic membrane. A thorough inspection of her teeth revealed excessive wear on the incisal edges, and the cause of her otalgia was identified as bruxism-related temporomandibular joint disorder. After the use of an occlusal splint and repeated botulinum toxin injections in the masseter and anterior temporalis muscles, along with good sleep hygiene, she experienced significant relief of pain and symptoms. The author suggests that multidisciplinary cooperation between ENT clinicians and dentists is necessary for the quick and accurate diagnosis and treatment of bruxism and the consequential referred otalgia.

J Audiol Otol 2016;20(2):123-126

KEY WORDS: Earache $\cdot$ Tooth wear $\cdot$ Teeth $\cdot$ Referred pain.

\section{Introduction}

Otalgia originating from a source outside the ear poses a difficult diagnostic challenge for even the most experienced otolaryngologist. In cases of a negative otologic exam and persistent otalgia, various factors could potentially be responsible for the pain, because the ear is innervated by many cranial and cervical nerves [1]. While a thorough history and physical examination provides the best clinical diagnostic assessment, otalgia due to dental disorders is often overlooked.

Bruxism is defined as a habitual, involuntary grinding or clenching of the teeth [2]. It is a form of dystonia characterized by hyperactivity of the muscles of mastication. Bruxism is a predisposing and exacerbating factor of temporomandibular joint disorders (TMDs), which are a common cause of secondary otalgia [3].

In this case report, the patient presented with a sharp and penetrating earache, which was found to be induced by bruxism. After dental treatment, her symptoms were alleviated. This highlights the need for multidisciplinary cooperation be-

This is an Open Access article distributed under the terms of the Creative Commons Attribution Non-Commercial License (http://creativecommons.org/licenses/by-nc/3.0/) which permits unrestricted non-commercial use, distribution, and reproduction in any medium, provided the original work is properly cited. tween ENT clinicians and dentists in the diagnosis and treatment of bruxism-induced otalgia.

\section{Case Report}

A 29-year-old female patient presented with a several-year history of intractable right-sided otalgia. She reported having no hearing loss, tinnitus, or dizziness. She described the pain as sharp and penetrating. The pain was intermittent and sometimes lasted for several hours. It varied in intensity throughout the day, and was usually worse in the morning. The otalgia frequently radiated to the right temporal area. She had received unsuccessful medical treatments for migraine headache. The otoendoscopic examination revealed a normal tympanic membrane without obvious otologic abnormalities (Fig. 1). The nasal endoscopy also showed normal findings without other signs of sinus inflammation. An examination of the oral cavity revealed no dental caries, pulpal inflammation of the posterior teeth, or other dental conditions that can cause ear pain. Her upper and lower third molars, which are known to cause otalgia when impacted, had been extracted 5 years ago. However, during a thorough inspection of the teeth, excessive wear on the incisal edges of the teeth resulting from end-to-end contact was observed (Fig. 2, 3). 


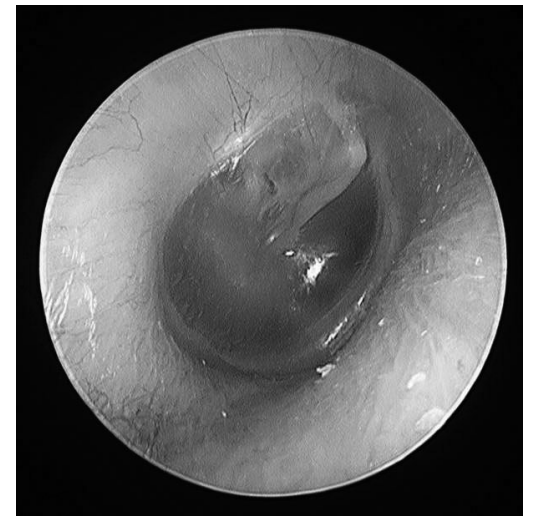

Fig. 1. Otoendoscopic examination showed a normal right tympanic membrane.

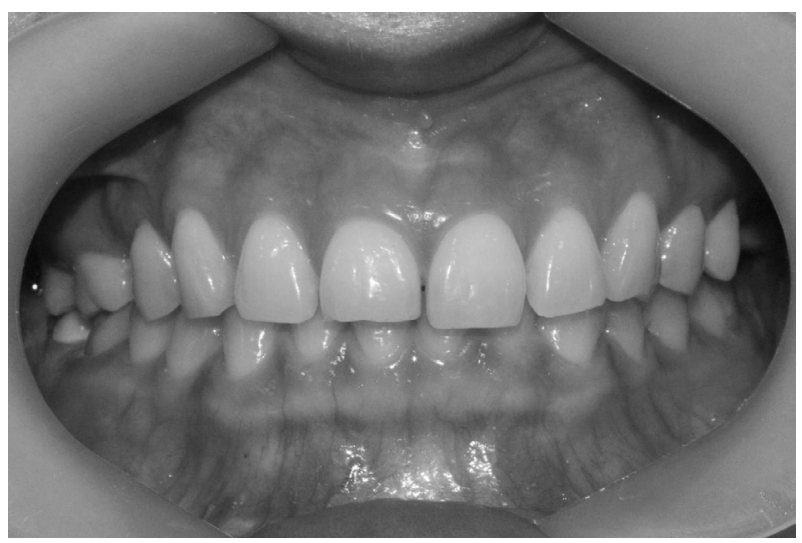

Fig. 2. Intraoral dental situation in occlusion, frontal view. Excessive wear and flattening of the cusp tips of the upper incisal edges was noted.

Moreover, her sleep partner reported hearing tooth grinding sounds, thus the cause of her otalgia was identified as bruxism-related TMD. The patient had a history of sleep bruxism and the presence of masseter muscle hypertrophy during voluntary contraction on her right side (Fig. 4). She was referred to a dentist and treated with an occlusal splint (Fig. 5) and local injections of botulinum toxin type A (BTX-A). The occlusal splint was composed of a hard acrylic resin and adjusted by the dentist once a month. The BTX treatment protocol included muscular administration of a total of $100 \mathrm{U}$ BTX-A (Botox; Allergan Inc., Irvine, CA, USA) in the masseter and anterior temporalis muscles bilaterally. The patient received monthly repeated BTX-A injections over 4 months. Self-massage therapy on the masseter and temporal muscles with sliding and kneading maneuvers was done repeatedly. The author instructed the patient on the practice of good sleep hygiene. This included the cessation of smoking and the intake of coffee or alcohol at night, limiting of physical or mental activity before bed, good bedroom conditions (quiet and dark), and the maintenance of regular sleep patterns. After 2 months, the patient experienced a significant relief of pain

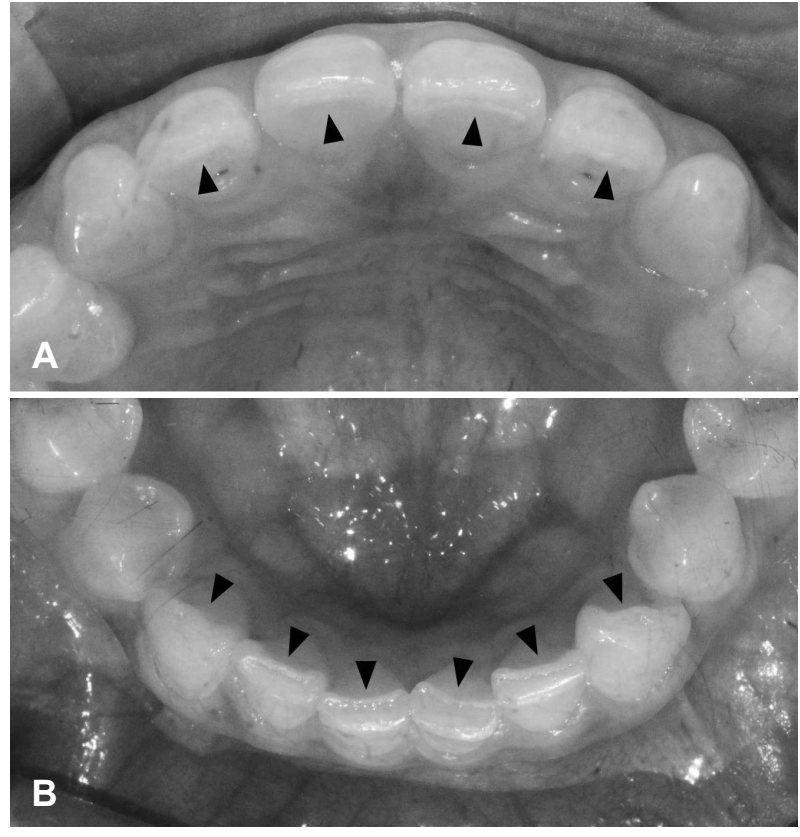

Fig. 3. Intraoral occlusal view. A: Upper teeth of the maxilla. B: Lower teeth of the mandible. Attrition on the incisal edges of the teeth from tooth-to-tooth contact (black arrowheads) was noted.

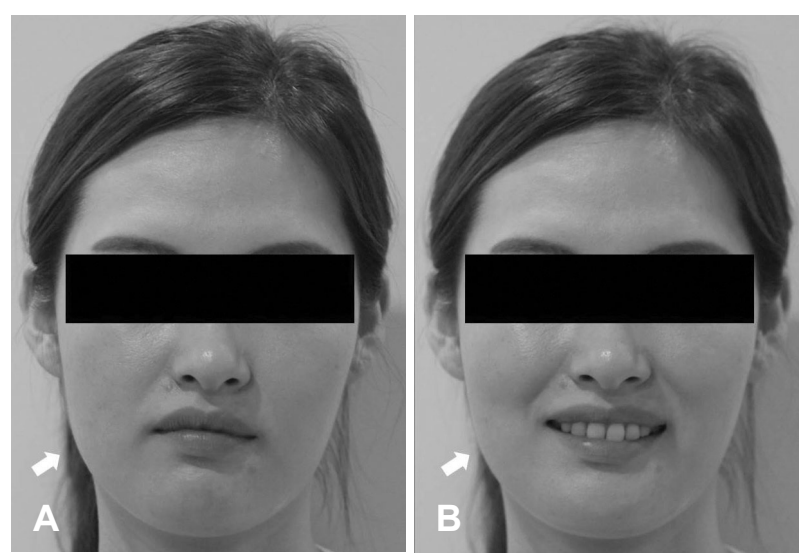

Fig. 4. Photographic findings show hypertrophy of the masseter muscle on the right side when the patient was $(A)$ clenching and (B) smiling (white arrows).

and symptoms. At a post-treatment follow up after 6 months, the significant relief was maintained.

\section{Discussion}

Otalgia can be classified as primary (otogenic, originating from pathologies inside the ear) or referred (nonotogenic, originating from outside the ear). A negative otologic exam and otalgia can be related to various diseases because the ear has a rich nerve supply from many cranial (V, VII, IX, and X) and cervical (C2 and $\mathrm{C} 3$ ). Referred otalgia often poses a difficult diagnostic challenge to otolaryngologists.

The term "bruxism" originates from the Greek word bry- 

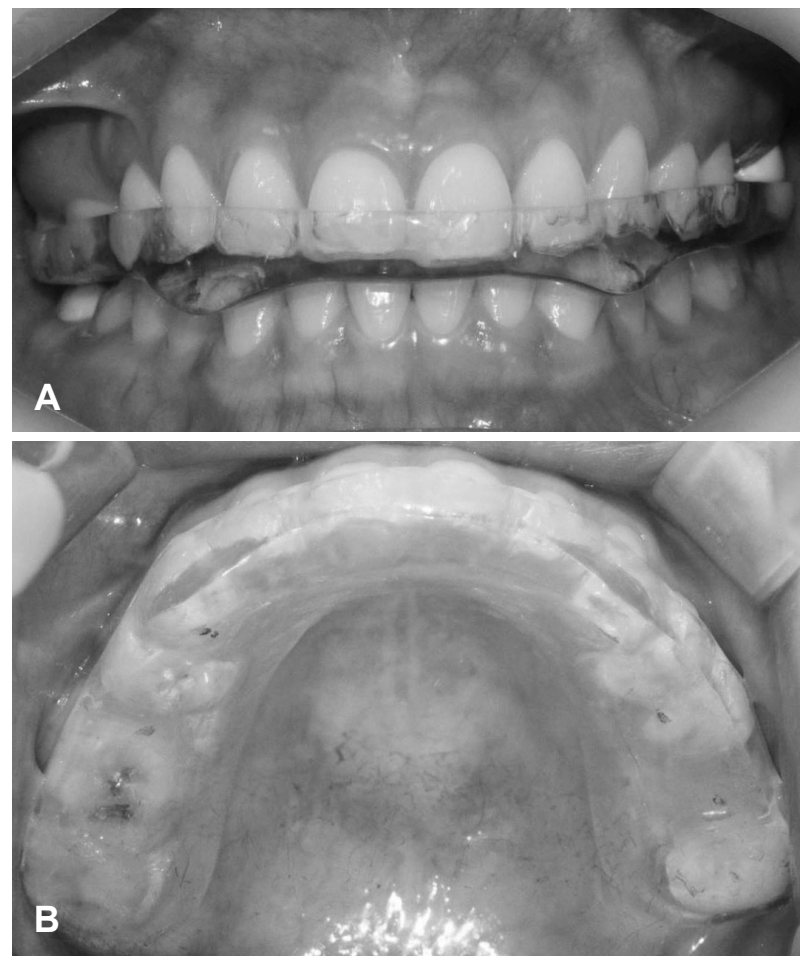

Fig. 5. A: Frontal view of the occlusal splint. B: The design of the splint covers the occlusal surfaces of the entire maxillary dental arch.

chein, which means to grind. Bruxism is generally defined as non-functional jaw movement characterized by the involuntary grinding and/or clenching of the teeth. It is thought to be an important etiological factor of abnormal tooth wear and periodontal disease. This disorder can also trigger headaches, TMDs, and pain in the masticatory muscles [4]. The prevalence of bruxism has not been well defined (ranging from $6-95 \%$ in previous studies) because of differences in the methods and criteria for evaluating bruxism [5].

The etiology of bruxism remains unclear despite the proposal of numerous theories. While it is evident that bruxism can destructively affect the oral structures and masticatory system, it is difficult to understand the condition and its associated activity clinically.

Bruxism can occur during wakefulness or sleep. The American Academy of Sleeping Disorders proposed the terms Awake and Sleep Bruxism. Bruxism during periods of wakefulness is 'Awake bruxism' (AB), otherwise termed diurnal bruxism. $A B$ is usually seen as a jaw clenching habit that appears in response to stress and anxiety [6]. On the other hand, bruxism during sleep, whether during the daytime or night, is termed 'Sleep bruxism' (SB), also known as nocturnal tooth grinding. SB is a sleep related rhythmic masticatory activity generally associated with periods of arousal [7]. Both $\mathrm{AB}$ and SB are sub-classified as either primary, not related to any oth- er medical condition, or secondary, associated with neurological disorders or an adverse side effect of drugs.

Bruxism can lead to dental wear, jaw muscle pain and fatigue, and temporal headaches. In some severe forms it can compromise oral functions such as chewing, speaking, and swallowing [8]. This is a predisposing and exacerbating factor of TMDs, which are a common cause of referred otalgia [3]. The most common otologic symptoms related to TMDs are otalgia, tinnitus, and vertigo [9].

Referred otalgia secondary to TMDs can be explained by the closely connected sensory innervation of the oral cavity, oropharynx, and ear, which are derived from the same embryological origins [10]. The ear is innervated by many nerves, including the fifth, seventh, ninth, and tenth cranial nerves, as well as superficial sensory branches from the cervical plexus. Similarly, the temporomandibular joint is innervated by the auriculotemporal branch of the fifth and seventh cranial nerves. The otologic symptoms that are commonly associated with TMDs, such as tinnitus, otalgia, dizziness, and/or vertigo, are also the most commonly observable effects of bruxism [11]. These otologic symptoms are known to be improved by dental treatment and therefore it is important to differentiate between primary otologic symptoms and those caused by bruxism.

In real clinical practice, TMDs are sometimes misdiagnosed as tension headaches or migraine because of the nature of the headache. Otolaryngologists and neurologists can easily overlook these disorders and conduct unnecessary and expensive investigations. In the present case, the patient presented with intermittent and intractable right otalgia frequently radiating to the ipsilateral temporal area. She had been taking a migraine-specific medication and other analgesic drugs. In cases such as this, the patient may pay multiple visits to otorhinolaryngology, neurology, and pain management clinics, where the examinations reveal normal findings.

The treatments for bruxism include intraoral appliances, medications, behavioral modification techniques, and injections of botulinum toxin in the masticatory muscles.

The use of an occlusal splint is considered a conservative and reversible therapy for patients with TMDs and/or bruxism to prevent tooth attrition and overload of the temporomandibular joint structures and masticatory muscles [12]. In general, the design of the device is simple, it covers the whole maxillary or mandibular dental arch, and is well tolerated by patients. In the present case, the patient showed severe wear on the anterior teeth. Additionally, the nocturnal occlusal force generated during the sleep-associated bruxism was so strong that the occlusal splint was frequently fractured. She was asked to use the occlusal splint continuously, in order to 
protect her teeth even while asleep.

Intramuscular injections with BTX are used in the treatment of the movement disorders associated with increased muscle tone, such as spasticity and dystonia, and the autonomic disorders associated with cholinergic overactivity and hyperhidrosis. Because of its muscle-relaxing and possible analgesic effects, BTX has also gained much interest as a possible treatment for myofascial pain and headache disorders [13]. Many studies have reported that BTX injections are effective for controlling AB. Other studies with only SB patients showed that BTX injections can reduce the frequency of jaw motor events [14], decrease bruxism-induced pain levels, and are equally effective as oral splints for alleviating pain [15]. The bulky region of the masseter and temporalis muscles has been regarded as an effective point for BTX injections, as it is the richest arborized area of the perforating nerve branches. The effects of a single BTX injection on the masticatory force are known to last from 2 weeks to up to 24 weeks, but start to decline 4 weeks after injection. In the present case, the patient received monthly repeated BTX-A injections over 4 months in the masseter and anterior temporalis muscles bilaterally in order to reduce the sustained force between the opposing teeth.

No studies have been published on the relationship between good sleep hygiene and the extent of SB. However, $\mathrm{SB}$ is known to occur mainly in the lighter sleep stages, or 'rapid eye movement sleep'. Avoiding stimulants such as tea, coffee, alcohol, and cigarettes for several hours before bed and maintaining a regular sleep schedule promotes better sleep. Better sleep means that more time is spent in the deeper sleep stages, also known as 'slow wave sleep', and fewer periods of arousal occur.

After the treatment with the occlusal splint and BTX injections, along with good sleep hygiene, the symptoms of this patient were alleviated. Therefore, cooperation between ENT clinicians and dentists is required for the diagnosis and treatment of bruxism and the consequential referred otalgia.

In conclusion, the author recommends that ENT clinicians perform a rapid and simple dental examination in cases of a negative otologic exam and otalgia. The early diagnosis of bruxism is possible if ENT clinicians pay attention to the typical features: worn incisal edges, flattened occlusal surfaces of the molar teeth, scalloping of the lateral borders of the tongue, ridging of the buccal mucosa at the occlusal line, and masseter muscle hypertrophy. This practice will reduce the risk of misdiagnosis with other neurological causes such as migraine, and facilitate the timely and accurate diagnosis of referred otalgia secondary to bruxism.
In conclusion, referred otalgia secondary to bruxism can be a diagnostic challenge for otolaryngologists. Although the management of these disorders has traditionally fallen under the purview of dentists, the most common symptoms are otologic problems. Therefore, for patients presenting with a negative otologic exam and persistent otalgia, careful attention should be paid to their dental status, because of the potential of bruxism-induced otalgia. This would require multidisciplinary cooperation between ENT clinicians and dentists. The author determined that otalgia originating from bruxism deserves careful attention, along with the consideration of dental treatment, which will be helpful in eliminating bruxism-induced otalgia.

\section{Conflicts of interest}

The author has no financial conflicts of interest.

\section{REFERENCES}

1) Kim SH, Kim TH, Byun JY, Park MS, Yeo SG. Clinical differences in types of otalgia. J Audiol Otol 2015;19:34-8.

2) Kesikburun S, Alaca R, Aras B, Tuğcu I, Tan AK. Botulinum toxin injection for bruxism associated with brain injury: case report. J Rehabil Res Dev 2014;51:661-4.

3) Ellis E, Hupp JR, Tucker MR. Contemporary oral \& maxillofacial surgery. 4th ed. St. Louis: Mosby;2003.

4) Gomes CA, El-Hage Y, Amaral AP, Herpich CM, Politti F, KalilBussadori S, et al. Effects of massage therapy and occlusal splint usage on quality of life and pain in individuals with sleep bruxism: a randomized controlled trial. J Jpn Phys Ther Assoc 2015;18:1-6.

5) Koyano K, Tsukiyama Y, Ichiki R, Kuwata T. Assessment of bruxism in the clinic. J Oral Rehabil 2008;35:495-508.

6) Manfredini D, Lobbezoo F. Role of psychosocial factors in the etiology of bruxism. J Orofac Pain 2009;23:153-66.

7) Kato T, Rompré P, Montplaisir JY, Sessle BJ, Lavigne GJ. Sleep bruxism: an oromotor activity secondary to micro-arousal. J Dent Res 2001;80:1940-4.

8) Tan EK, Jankovic J, Ondo W. Bruxism in Huntington's disease. Mov Disord 2000;15:171-3.

9) Kim DS, Cheang P, Dover S, Drake-Lee AB. Dental otalgia. J Laryngol Otol 2007;121:1129-34.

10) Ely JW, Hansen MR, Clark EC. Diagnosis of ear pain. Am Fam Physician 2008;77:621-8.

11) Kitsoulis P, Marini A, Iliou K, Galani V, Zimpis A, Kanavaros P, et al. Signs and symptoms of temporomandibular joint disorders related to the degree of mouth opening and hearing loss. BMC Ear Nose Throat Disord 2011;11:5.

12) Barão VA, Gallo AK, Zuim PR, Garcia AR, Assunção WG. Effect of occlusal splint treatment on the temperature of different muscles in patients with TMD. J Prosthodont Res 2011;55:19-23.

13) Belvis R, Mas N. Treatment of chronic migraine with intramuscular pericranial injections of onabotulinumtoxin a. Recent Pat CNS Drug Discov 2014;9:181-92.

14) Guarda-Nardini L, Manfredini D, Salamone M, Salmaso L, Tonello $\mathrm{S}$, Ferronato G. Efficacy of botulinum toxin in treating myofascial pain in bruxers: a controlled placebo pilot study. Cranio 2008;26: 126-35.

15) Long H, Liao Z, Wang Y, Liao L, Lai W. Efficacy of botulinum toxins on bruxism: an evidence-based review. Int Dent J 2012;62:1-5. 